\title{
Radial palsy in an individual with high-level chronic spinal cord injury
}

\author{
Nathan Hitchman ${ }^{1,2} \cdot$ Heather Finlayson ${ }^{3,4} \cdot$ Andrei Krassioukov $^{1,3,4}$
}

Received: 3 May 2020 / Revised: 13 June 2020 / Accepted: 14 June 2020

(C) International Spinal Cord Society 2020

\begin{abstract}
Introduction Prolonged compression is a common cause of radial neuropathy in able-bodied individuals but has not been reported in individuals with chronic SCI. This is despite the fact that individuals with SCI may be at increased risk of peripheral nerve injuries due to wheelchair mobility and baseline sensory deficits. Furthermore, diagnosis of peripheral nerve injury poses a unique challenge in this population because symptoms and signs are superimposed on pre-existing central deficits.

Case presentation We present the case of a 48-year-old man with a C6 AIS A SCI from a motor vehicle accident 22 years earlier who had a new onset compressive radial neuropathy. At initial assessment he complained of paresthesia along his lateral right arm accompanied by new onset wrist-drop. Subsequent radial nerve conduction studies revealed severe reductions in amplitude for sensory and motor action potentials. The patient was managed with mobility exercises and vitamin B supplementation and showed full recovery of motor and sensory function to baseline levels on follow-up 4 months after the injury. Discussion The electrophysiologic profile of this patient is illustrative of severe nerve compression for an extended time period. Unlike able-bodied individuals who can reposition themselves to alleviate nerve compression, individuals with SCI may be unaware of nerve compression or unable to reposition themselves. This highlights the need for precautionary measures such as maneuvers and devices to provide trunk and limb stability along with the use of medical alert devices that allow individuals to access timely help when unattended.
\end{abstract}

\section{Introduction}

Prolonged compression is a common cause of radial neuropathy [1]. The ensuing pathology — termed "Saturday night palsy" after its classical presentation in inebriated individuals who fall asleep with an arm over a chair-has been well described in otherwise able-bodied individuals but has not been reported in individuals with chronic spinal

Andrei Krassioukov

andrei.krassioukov@vch.ca

1 International Collaboration on Repair Discoveries, University of British Columbia, Vancouver, BC, Canada

2 MD Undergraduate Program, University of British Columbia, Vancouver, BC, Canada

3 Faculty of Medicine, Division of Physical Medicine and Rehabilitation, University of British Columbia, Vancouver, BC, Canada

4 GF Strong Rehabilitation Centre, Vancouver Coastal Health, Vancouver, BC, Canada cord injury (SCI). This is despite the fact that spinal cordinjured individuals may be at increased risk of these and other peripheral nerve injuries due to wheelchair mobility and transfers, and because of sensory deficits [2]. Furthermore, late diagnosis and under-diagnosis of such injuries is more likely in this population given that signs and symptoms are superimposed on pre-existing deficits related to chronic SCI [2]. We describe the first reported case of acute compressive radial neuropathy in a tetraplegic patient, including clinical and electrophysiologic features and course of recovery.

\section{Case presentation}

A 48-year-old man with C6 American Spinal Injury Association Impairment Scale [3] A SCI from an accident 22 years earlier presented to the clinic 1 week after an incident that resulted in prolonged radial nerve compression. In this incident he fell forward and became constrained partially out of his motorized wheelchair and against a wall with his torso in a hyperflexed position and his right knee forcibly 
pressed into his right axilla. Due to his position between the wheelchair and the wall he was unable to move himself to alleviate the pressure to his right axilla, and he fell asleep until a care aid arrived $7 \mathrm{~h}$ later.

At first assessment he complained of pain and paresthesia along the lateral aspect of his right arm from axilla to wrist, accompanied by new wrist extensor weakness. Clinical examination showed a new wrist-drop (MRC grade 2/5; previous baseline 4/5). This wrist-drop of his dominant hand prevented him from operating the controls of his motorized wheelchair, thereby severely limiting his mobility and activities of daily living, which he previously performed independently. Elbow extension and arm abduction were preserved at MRC grade of 2/5 and 5/5 respectively, similar to his baseline values. The last normal sensory level was C5, with no pinprick sensation at the C6 dermatome, the same as his previous baseline. The left arm was unaffected by this incident.

The patient was managed with mobility exercises and vitamin B supplementation and was followed-up for clinical examination and electrophysiologic testing at

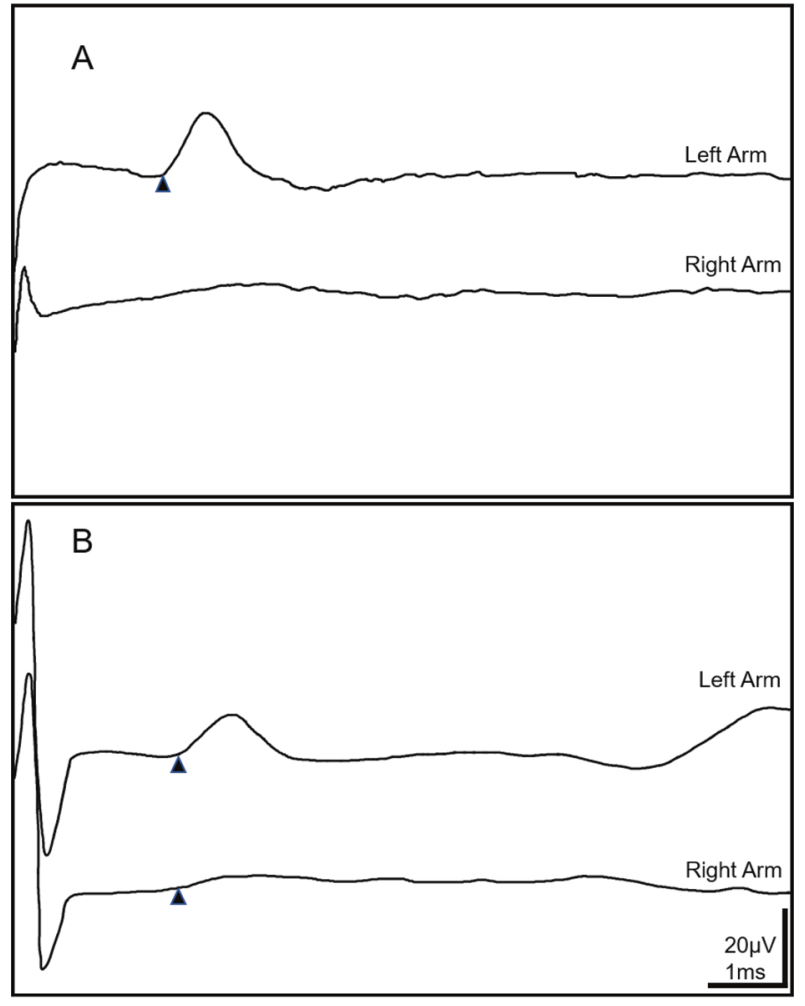

Fig. 1 Radial nerve conduction studies. SNAP measured 1 month (a) and 9 months (b) after compressive injury. Tracings for the unaffected left arm are included for comparison. Arrows indicate the beginning of a SNAP, where detectable. At 1-month post injury forearm-snuffbox superficial radial nerve SNAP amplitude was reduced in the affected right arm $(2.8 \mathrm{mcV})$ compared to a normal value in the left arm $(17 \mathrm{mcV})$. After 9 months, this had improved $(6.3 \mathrm{mcV})$ but was still considerably lower than the left arm $(18.2 \mathrm{mcV})$, demonstrating only partial recovery. Latency and conduction velocity were normal for all studies. approximately 1 month after injury. At this time sensory abnormalities secondary to this injury had fully recovered and wrist extension had improved significantly but not completely (MRC grade 4-/5). Nerve conduction studies of the right superficial radial nerve revealed a severe reduction in amplitude to $2.8 \mathrm{mcV}$ (left arm normal, $17 \mathrm{mcV}$; Fig. 1). Latency and conduction velocity were normal. The right median finger 2 response had a normal amplitude, latency, and conduction velocity, indicating sparing of the median nerve consistent with the clinical presentation. Needle electromyography (EMG) of the right triceps and extensor carpi radialis longus revealed active denervation changes with positive sharp waves. In the right extensor carpi radialis longus, motor unit amplitudes were increased, and the interference pattern was reduced with rapid firing. The patient had difficulty voluntarily activating the right triceps. EMG of the right biceps was normal.

At follow-up evaluation 4 months after initial onset of the right wrist weakness, the patient reported complete recovery of sensory and motor function to his baseline levels, which was confirmed on clinical examination. Electrophysiologic tests were repeated at 9 months post injury. These demonstrated improved right radial nerve function. Specifically, forearm-snuffbox superficial radial sensory nerve action potential (SNAP) amplitude had increased from $2.8 \mathrm{mcV}$ at 1-month post injury to $6.3 \mathrm{mcV}$ (Fig. 1).

\section{Discussion}

Diagnosis of peripheral nerve injuries in patients with chronic SCI is challenging because symptoms and signs are superimposed on pre-existing deficits related to SCI (e.g. decrease of sensation, presence of paresthesias etc.). Existing literature suggests that such injuries are underdiagnosed when occurring at the time of SCI but it is not known whether this holds true for individuals with preexisting chronic SCI [4]. Diagnosis of peripheral nerve injury in our patient was aided by the existence of an obvious traumatic incident with subsequent new symptoms. Whereas localization of such an injury in able-bodied individuals is relatively simple based on the distribution of sensory and motor deficits, it was complicated in our patient by his total lack of sensation below the $\mathrm{C} 5$ dermatome and diminished voluntary motor function below the level of $\mathrm{C} 8$.

The combination of right wrist extensor weakness (MRC grade 2/5; baseline MRC grade 4/5) and paresthesia along the lateral aspect of the forearm implicated the radial nerve. This, taken together with sparing of deltoid power (MRC grade 5/5), ruled out injury proximal to the branching of the axillary nerve, namely, brachial plexus injury. In ablebodied patients, radial nerve injury can be further localized as being proximal to the spiral groove of the humerus, in 
which case triceps power is affected, or distal to it, in which case triceps power is spared [5]. In light of our patient's reduced baseline triceps power (MRC grade 2/5), the finding of relatively unchanged function in this area was ambiguous.

Electrophysiologic testing confirmed the diagnosis of radial nerve injury. However, nerve conduction studies of our patient demonstrated severe reductions in amplitude with normal conduction velocity and latency. This contrasts with studies of Saturday night palsy in able-bodied individuals, which report the opposite pattern: reduced conduction velocity and increased latency as dominant features, with reduction in amplitude occurring in only a small minority of patients [6-8]. This commonly reported constellation of electrophysiologic features is representative of focal demyelination, which is thought to be the dominant pathology in compressive neuropathy [9].

The dissimilarity of our patient's electrophysiologic profile to this literature may represent a difference in injury mechanism. Animal experiments show that mild, short duration compression produces slowed conduction, whereas longer and more severe compression results in conduction block, manifested as reduced action potential amplitude [10]. This paradoxical sparing of conduction velocity in more severe compressive injuries can be explained by the fact that large diameter fast-conducting fibers are the most susceptible to compressive injury [10]. Thus, mild compression may lead to differential demyelination of fast-conducting fibers and sparing of slower-conducting small fibers, resulting in the appearance of slowed conduction velocity. However, with more severe compressive injury all fiber types are affected equally, resulting in conduction block of individual neurons but little or no reduction in net conduction velocity [11]. This explanation fits the mechanism of injury for our patient, who experienced compression of longer duration than most reported cases of Saturday night palsy in able-bodied patients and whose forward-leaning position would likely have exerted greater pressure on the radial nerve than is typically seen in this type of injury [7].

This points to what may be an important difference between able-bodied and chronic SCI patients with respect to compressive nerve injury. Unlike able-bodied individuals who can reposition themselves in the event of nerve compression, individuals immobilized by SCI may be unable to do so until a care provider is present, as was the case with our patient. Whereas the average duration of compression for able-bodied individuals with Saturday night palsy has been reported to be about $3 \mathrm{~h}$, ours is the first report of this injury in a patient with chronic SCI and he experienced compression for 7 hours before receiving assistance [7].

This incident highlights the need for precautionary measures in this population. This may include the combination of maneuvers and devices to provide trunk and limb stability and medical alert devices that allow individuals to access timely help. The latter is especially important for reducing morbidity given that duration of compression is directly linked to severity of injury and prognosis [10].

Acknowledgements We thank Shane Balthazaar for his critical input into this case report.

Funding $\mathrm{NH}$ was funded by the University of British Columbia Summer Student Research Project program.

\section{Compliance with ethical standards}

Conflict of interest The authors declare that they have no conflict of interest.

Publisher's note Springer Nature remains neutral with regard to jurisdictional claims in published maps and institutional affiliations.

\section{References}

1. Duz B, Solmaz I, Civelek E, Onal MB, Pusat S, Daneyemez M. Analysis of proximal radial nerve injury in the arm. Neurol India 2010;58:230.

2. Bursell JP, Little JW, Stiens SA. Electrodiagnosis in spinal cord injured persons with new weakness or sensory loss: central and peripheral etiologies. Arch Phys Med Rehab. 1999;80:904-9.

3. Kirshblum SC, Burns SP, Biering-Sorensen F, Donovan W, Graves DE, Jha A, et al. International standards for neurological classification of spinal cord injury (revised 2011). J Spinal Cord Med. 2011;34:535-46.

4. Rhee PC, Pirola E, Hébert-Blouin MN, Kircher MF, Spinner RJ, Bishop AT, et al. Concomitant traumatic spinal cord and brachial plexus injuries in adult patients. J Bone Joint Surg Am. 2011;93:2271-7.

5. Kimbrough DA, Mehta K, Wissman RD. Case of the season: Saturday Night Palsy. Semin Roentgenol. 2013;48:108.

6. Arnold WD, Krishna VR, Freimer M, Kissel JT, Elsheikh B. Prognosis of acute compressive radial neuropathy. Muscle Nerve. 2012;45:893-4.

7. Han BR, Cho YJ, Yang JS, Kang SH, Choi HJ. Clinical features of wrist drop caused by compressive radial neuropathy and its anatomical considerations. J Korean Neurosurg Soc. 2014;55:148.

8. Suh BC, Kim B, Kim Y, Chung P, Moon H, Yoon WT, et al. Diagnostic and prognostic usefulness of proximal radial motor nerve conduction study in acute compressive radial neuropathy. Clin Neurophysiol. 2012;123:e19

9. Trojaborg W. Rate of recovery in motor and sensory fibres of the radial nerve: clinical and electrophysiological aspects. J Neurol, Neurosurg Psychiatry. 1970;33:625-38.

10. Ochoa J, Fowler TJ, Gilliatt RW. Anatomical changes in peripheral nerves compressed by a pneumatic tourniquet. J Anat. 1972;113:433.

11. Fowler TJ, Danta G, Gilliatt RW. Recovery of nerve conduction after a pneumatic tourniquet: observations on the hind-limb of the baboon. J Neurol, Neurosurg Psychiatry. 1972;35:638-47. 